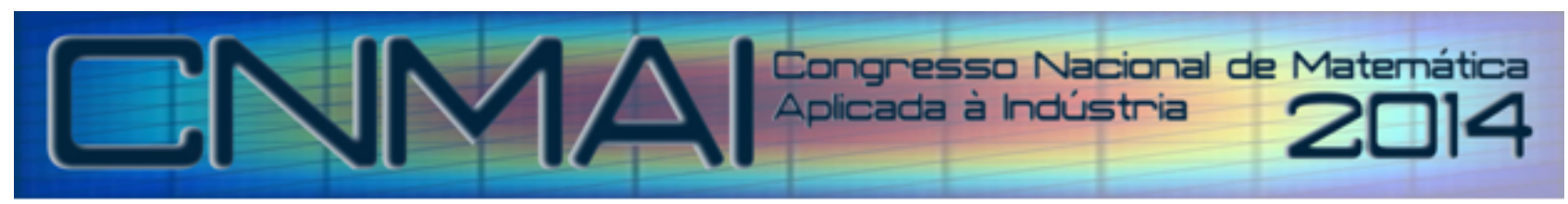

18 a 21 de novembro de 2014, Caldas Novas - Goiás

\title{
ESTUDO E DESENVOLVIMENTO DE SIMULADORES DE ATERRISSAGEM DE AERONAVES UTILIZANDO LÓGICA FUZZY.
}

\author{
Rafael de Oliveira Taveira, rottaveira@gmail.com ${ }^{1}$ \\ Sergio Francisco da Silva, sergio.f. silva@gmail. $\mathrm{com}^{1}$
}

${ }^{1}$ Universidade Federal de Goiás, Av. Dr. Lamartine Pinto de Avelar, 1120, St.Universitário, Catalão - GO

\begin{abstract}
Resumo. A noção de conjuntos fuzzy foi concebida em 1965, o foco era estabelecer um meio de tratar problemas de caráter subjetivo com informações vagas e imprecisas de maneira formal e também lidar com problemas nos quais há dados numéricos e conhecimento na forma linguística. Baseado na teoria de lógica fuzzy, este trabalho tem por objetivo apresentar um sistema automático de pouso para aeronaves, que pode ser denominado como um meio para guiar e controlar uma aeronave automaticamente a partir de uma estimação inicial de altitude para um ponto onde um contato seguro é feito com a superfície de pouso. Esses sistemas fornecem não só orientação como também fornecem o controle da aeronave, incluindo informações sobre a velocidade e a posição da aeronave em relação ao terreno abaixo dela.
\end{abstract}

Palavras-chave: fuzzy, aeronaves, aterrissagem

\section{INTRODUÇÃO}

Este artigo tem por objetivo principal implementar um sistema de piloto automático para pouso (Automatic Landing System). Os ALS tiveram início na Grã-Bretanha adotados pela Royal Air Force em meados da década de 40 (Fuller, 2012) onde a baixa visibilidade ocasionada pelo inverno europeu e o considerável aumento da poluição industrial resultava em muitos acidentes na fase de aterrissagem. O desenvolvimento prosseguiu e em 1955 esta tecnologia foi adotada pela International Civil Aviation Organization (ICAO) para uso no aeródromo civil da Grã-Bretanha e no exterior. Atualmente várias pesquisas visam aprimorar o desempenho de um ALS utilizando técnicas de Inteligência Artificial (Boskoski et al., 2005; McLauchlan, 2009; Lakovic e Lotinac, 2010; Raj e Tattikota, 2013), essas pesquisas visam aperfeiçoar o processamento dos dados recebidos pelo Instrument Landing System (ILS) para melhorar a qualidade do pouso em diversas situações difíceis, como por exemplo, falta de visibilidade, tempestades e vórtices de ventos. Segundo um levantamento da empresa Boeing divulgado em agosto de 2013 com dados de desastres aéreos de 1959 a 2012, 57\% dos acidentes ocorrem no decorrer da fase de aterrissagem (Boeing, 2013), durante essa fase, o avião está mais próximo do chão e mais vulnerável a falhas. No momento da aterrissagem, as falhas mais comuns são humanas, dado que a tripulação está sob maior pressão psicológica e estresse e tem menos tempo de decisão para manobras de emergência, o que resulta em um momento crítico, pois qualquer falha pode dar início a uma sequência de erros que eventualmente causam um acidente fatal. Neste projeto, utilizaremos um sistema de inferências fuzzy que propõe uma trajetória para o controle não linear da aterrissagem do avião. Essa abordagem é útil pois esse sistema é capaz de expressar um raciocínio aproximado que pode ser utilizado na tomada de decisões em tempo real, evitando assim erros comuns que ocasionam graves acidentes. A lógica fuzzy é uma ferramenta ideal para este tipo de situação pois tem se mostrado mais adequada para tratar informações imprecisas através de uma base de conhecimento, no qual está em formato de regras de produção, fáceis de examinar e entender. Este formato de regras torna mais fácil a manutenção e a atualização da base de conhecimento. Na seção 2 explicaremos o conceito de aterrissagem de aeronaves, na seção 3 conceituaremos a lógica fuzzy, na seção 4 descreveremos a métrica utilizada para este projeto e seu desenvolvimento. Os resultados são apresentados na seção 5 e apresentaremos nossas conclusões na seção 6.

\section{DESCRIÇÃO DO PROBLEMA}

O controle da trajetória de descida de uma aeronave é uma das tarefas mais difíceis para um piloto automático, pois exige controle simultâneo de diversas variáveis: velocidade, ângulo de trajetória, ângulo de ataque, altitude, etc., que é importante tanto em termos de estabilidade da aeronave como em performance. 
Segundo Paglione (1985), a trajetória de pouso pode ser dividida em 4 etapas: espera (altitude-hold), aproximação (glideslope), arredondamento (flare) e rolamento (landing roll), como ilustrado na Figura 1. O foco deste artigo está na fase de aproximação e arredondamento, que representa o início do processo de aterrissagem onde os flapes estão defletidos de maneira a obter o coeficiente de sustentação e com isso reduzir a velocidade de aproximação. Nessa etapa a trajetória se torna uma reta com determinado ângulo de declive determinada pelo ILS. O arredondamento inicia quando a altitude da aeronave alcança um valor especificado que possibilita uma curvatura na trajetória, para que, desta forma, a aeronave tenha um toque suave na pista e um bom rolamento.

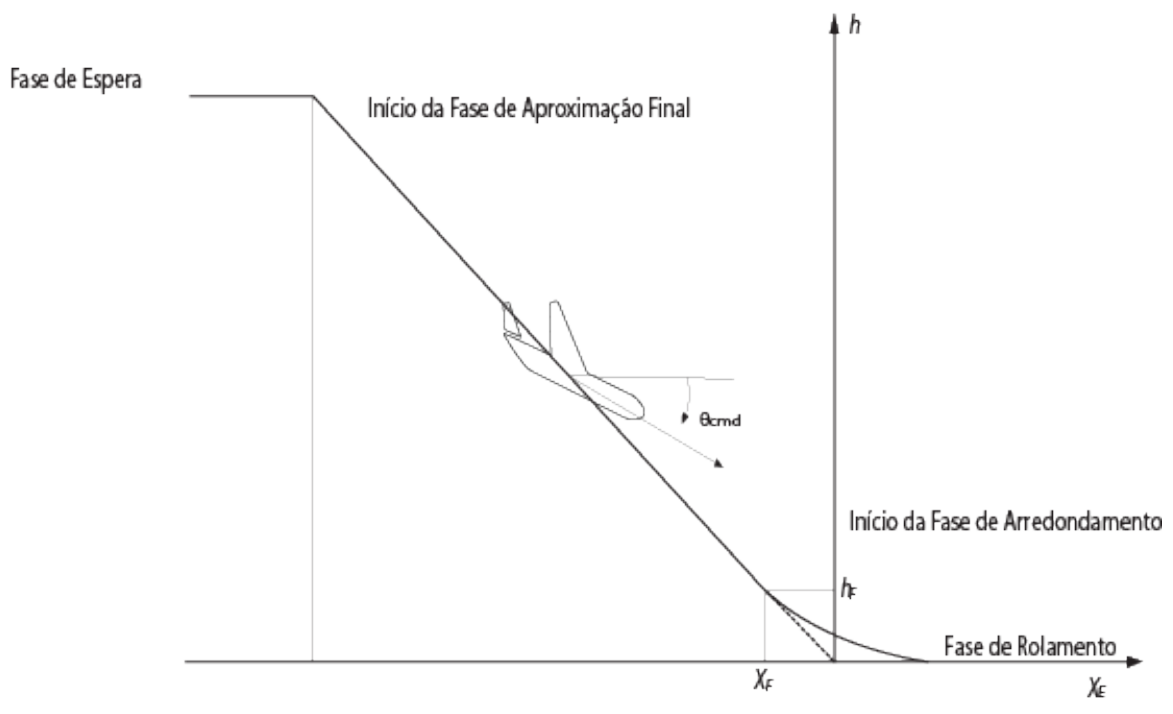

Figura 1. Fase de aproximação e arredondamento

Quando encontrado um ângulo de arfagem apropriado, um correto alinhamento com a pista de pouso e uma velocidade adequada, perfeitamente alinhada ao ILS temos o glide path, que representa o caminho adequado que a aeronave deve percorrer para uma correta aterrissagem, ou seja, caracteriza-se como uma transição suave do nível de vôo para o ângulo ou trajetória desejada.

\section{LÓGICA FUZZY}

A noção de conjuntos fuzzy foi concebida por Zadeh (1965), a ideia era estabelecer um meio de tratar problemas de caráter subjetivo com informações vagas e imprecisas de maneira formal e também lidar com problemas nos quais há dados numéricos e conhecimento na forma linguística, a ideia básica da lógica fuzzy é imitar o controle das ações similarmente a um ser humano.

Um conjunto fuzzy é caracterizado por uma função de pertinência que associa a cada elemento do universo de discurso um número no intervalo real [0,1], a partir dessa função de pertinência podemos dizer o quanto determinado elemento pertence ao seu universo de discurso. A teoria de conjuntos é de fato o elemento chave para formulação das inferências fuzzy. A teoria de conjuntos fuzzy é baseada no fato de que os conjuntos existentes no mundo real não possuem limites precisos pois um conjunto fuzzy é um agrupamento impreciso, onde a transição de não-pertinência para pertinência é gradual, não abrupta.

Desta forma a lógica fuzzy se distancia da lógica clássica, também conhecida como lógica Aristotélica, onde determinado elemento tem caráter binário (bivalente): o elemento pertence $(x \in A)$ ou não pertence $(x \notin A)$ ao conjunto.

\subsection{Variáveis Linguísticas}

Uma variável linguística $u$ no universo de discurso, é definida como um nome (ex: temperatura) ou possíveis valores fuzzy (ex: frio, quente, nem frio nem quente) os quais são rótulos de conjuntos fuzzy que podem ser vagos para uma máquina, mas que expressam bem o conhecimento humano.

\subsection{Relações Crisp}

O produto cartesiano de dois conjuntos é determinado por: $X \times Y=\{(x, y) \mid x \in X, y \in Y\}$. A relação crisp $\mu_{B}(x, y)$ é dada por:

$$
\mu_{B}(x, y)=\{1,(x, y) \in X \times Y \text { ou } 0,(x, y) \notin X \times Y\}
$$

Onde 1 representa que existe relação e 0 representa que não existe relação. Se os conjuntos forem finitos será representado por uma matriz $R$ chamada de matriz de relação. 


\subsection{Regras de Base}

O conhecimento em geral é convenientemente expressado pela linguagem natural. A regra de base é uma maneira de representar o conhecimento utilizando a linguagem natural. Uma forma genérica da regra de base é:

Se (Premissa) Então (Consequente ou Conclusão)

A primeira parte da regra especifica as condições, chamadas premissas ou antecedentes. A segunda parte especifica as ações, chamadas conclusões ou consequentes. É tipicamente expressada como uma inferência de que, se é conhecido um fato, pode-se interferir ou derivar outro fato.

As informações fuzzy podem ser representadas na forma de regras de base, por exemplo:

Regra 1: SE $x=A$ ENTÃO $y=B$, onde $A$ e $B$ são conjuntos fuzzy.

Agora, considerando uma nova regra:

$$
\text { Regra 2: SE } x=A^{\prime} \text { ENTÃO } y=B^{\prime}
$$

A partir da regra 1 deriva-se a regra 2. O consequente $B^{\prime}$ pode ser encontrado na composição $B^{\prime}=A^{\prime} \circ R$, onde $R$ é uma matriz de relação.

\subsection{Sistema de Inferência $f u z z y$}

Suponhamos a classificação das pessoas pela sua altura (Jang et al., 1997), se considerarmos uma abordagem clássica, poderíamos afirmar que indivíduos altos são aqueles que possuem estatura igual ou superior a $2 \mathrm{~m}$. Segundo essa abordagem, podemos afirmar que uma pessoa com $2.01 \mathrm{~m}$ é alta e que uma pessoa com $1.97 \mathrm{~m}$ não pertence ao conjunto das pessoas altas. Percebe-se que esta abordagem não condiz com a realidade, contudo, utilizando a concepção de pertinência, pode-se atribuir valores decrescentes de pertinência a medida que a estatura reduz: $\mu_{A}(2)=1 ; \mu_{A}(1.95)=0.9 ; \mu_{A}(1.80)=0.6 ; \mu_{A}(1.70)=0.3$. Neste caso as pessoas com altura de $1.95 \mathrm{~m}$ teriam um alto grau de pertinência no conjunto das pessoas altas enquanto uma pessoa com $1.70 \mathrm{~m}$ teria uma pertinência menor neste conjunto.

Hoje, vários tipos de sistemas de inferência são utilizados (Behera e Kar, 2009). Nesta seção, nos referenciaremos a apenas um destes sistemas: o sistema Mamdani, o qual foi utilizado para a implementação deste projeto.

As entradas para o sistema de inferência são confusas, no entanto, estão presentes em determinado intervalo. Essas entradas são fuzzificadas utilizando funções de pertinência para que, desta forma, se aplique determinadas regras que utilizam um raciocínio aproximado com operadores lógicos $e(\wedge)$ e ou $(\vee)$.

A combinação destas regras é feita utilizando um processo de agregação. Neste projeto utilizaremos três tipos de agregação presentes nos sistemas Mamdani (MathWorks, 2010b):

- Máximo

- Probabilístico

- Soma

A saída, análogamente a entrada, deve ser também confusa, logo os dados gerados no processo de agregação devem ser desfuzzificados. Nesta etapa, utilizaremos três métodos presentes nos sistemas Mamdani (MathWorks, 2010b):

- Centro de Área (COA)

- Bisector

- Metade do Máximo (MOM)

A Figura 2 ilustra este processo, recebe-se uma entrada crisp que é imediatamente fuzzificada para que possa ser aplicada as regras de base através do sistema de inferência. Ao ser aplicada as regras, toda informação ainda está fuzzificada, então é entregue ao desfuzzificador para que possa ser transformada em uma saída com valores válidos para o sistema.

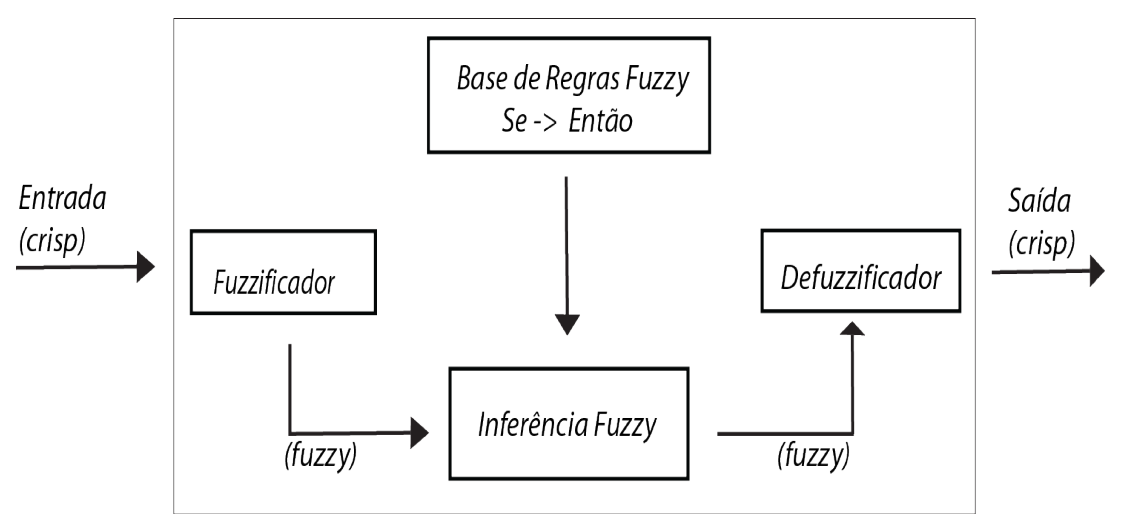

Figura 2. Sistema de inferência fuzzy 


\section{LÓGICA $F U Z Z Y$ E A ATERRISSAGEM DE AERONAVES}

O sistema fuzzy, com base nas informações fornecidas, inicialmente pelo ILS e, depois, pelo radar altímetro, especifica a trajetória de descida desejada para a aeronave, definindo os valores instantâneos desejados para a altitude e velocidade vertical (glide slope). Com base no erro entre os valores de referência e os valores reais, medidos pelo subsistema de navegação, o piloto automático determina os valores de ângulo de arfagem $\left(\Theta_{c m d}\right)$ e de velocidade da aeronave em relação ao ar (true airspeed), necessários para um pouso dentro dos limites aceitáveis. A saída de controle será uma força que, quando aplicada a uma aeronave, vai alterar a sua altura $(h)$ e a velocidade $(v)$.

\subsection{Controlador Fuzzy}

A lógica de tomada de decisão determina como as operações de lógica fuzzy são realizadas e, juntamente com uma base de conhecimento determina as saídas de cada regra do tipo Se-Então. Tais regras são combinadas e convertidas em valores precisos com o bloco de defuzzificação.

A fim de processar a entrada para obter a saída de raciocínio, existem seis passos envolvidos na criação de um sistema fuzzy baseado em regras:

1. Identificar as entradas e seus limites e nomeá-los.

2. Identificar as saídas e seus limites e nomeá-los.

3. Criar um grau de pertinência fuzzy para cada entrada e saída.

4. Construir a base de regras em que o sistema irá operar.

5. Decidir como a ação será executada através da composição entre as regras .

6. Combinar as regras e desfuzzificar a saída.

\subsection{Funções de Entrada e Saída}

Neste trabalho, utilizamos um sistema fuzzy com duas entradas e uma saída, onde a primeira entrada referencia a altura da aeronave em relação ao solo e a segunda entrada a velocidade vertical da aeronave, produzindo uma saída referente a força que, quando aplicada a aeronave, irá alterar tanto a altura quanto a velocidade vertical.

De maneira genérica, temos uma aeronave de massa $m$ movendo-se com velocidade $v$ em um dado momento $p$ $(p=m v)$. Se não existir uma força externa sendo aplicada, a massa $m$ continua na mesma direção com mesma velocidade $v$. No entanto, se ao longo de um intervalo de tempo $\Delta t$ uma força $F$ for aplicada, então teremos uma variação de velocidade proporcional a força aplicada.

Utilizando funções trapezoidais e triangulares (Behera e Kar, 2009), as Figura 3, Figura 4 e Figura 5 representam a pertinência para os conjuntos de entrada (velocidade e altura), e o conjunto de saída (força).

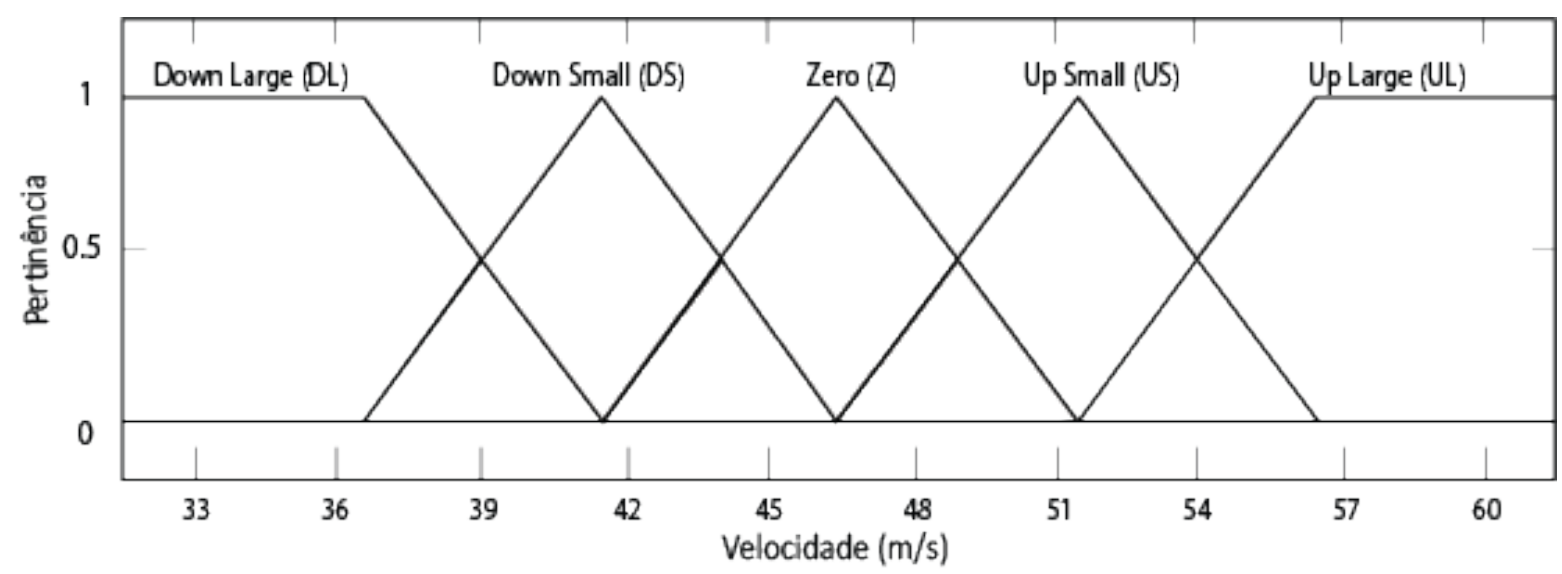

Figura 3. Função de pertinência para a velocidade 


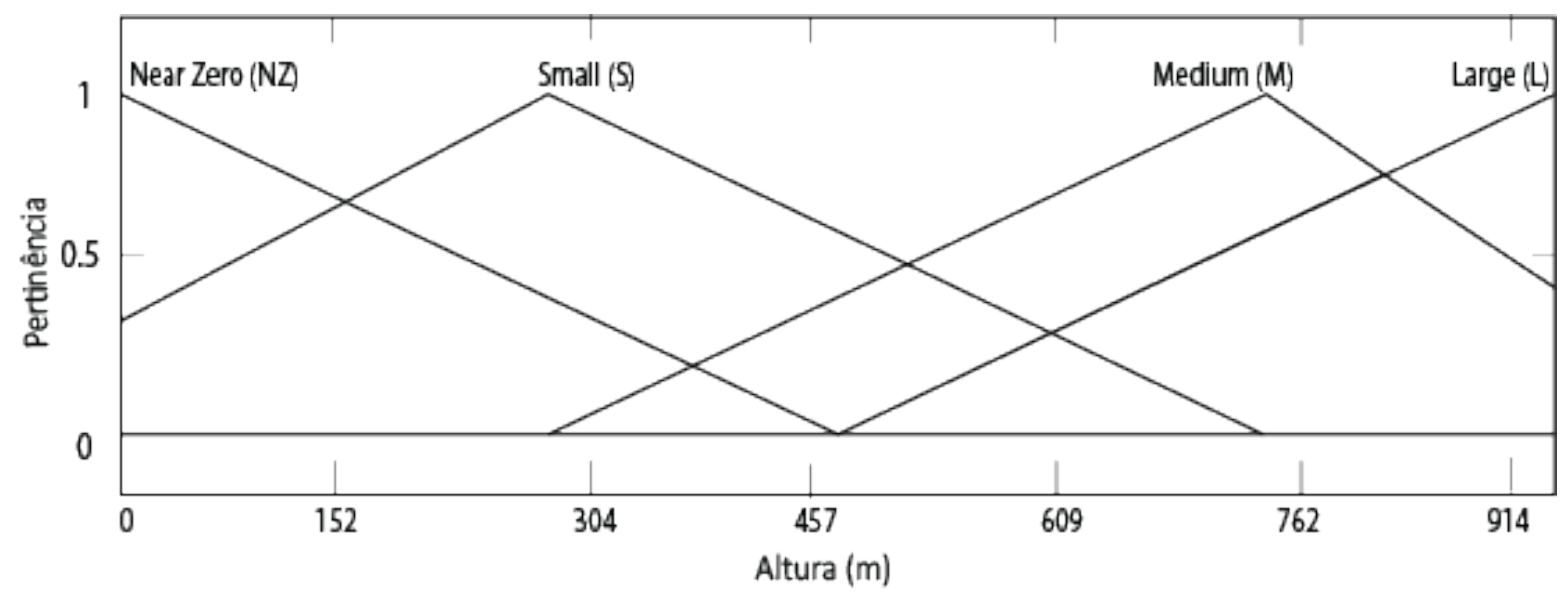

Figura 4. Função de pertinência para a altura



Figura 5. Função de pertinência para a força de saída

\subsection{Regras fuzzy}

Regras fuzzy são geralmente representadas utilizando uma matriz de relação entre os conjuntos. Para este problema temos uma matriz bi-dimensional com duas entradas (altura e velocidade) representada pela Tabela 1. Nesta matriz cada coluna representa uma função de pertinência para a velocidade vertical e cada linha representa uma função de pertinência para a altura. Os valores na matriz correspondem aos valores do conjunto de saída. Ao todo temos 20 regras, visto que temos quatro funções de pertinência para a altura e cinco funções de pertinência para a velocidade vertical.

Tabela 1. Matriz de relação

\begin{tabular}{|c|c|c|c|c|c|}
\hline \multirow{2}{*}{ Altura } & \multicolumn{5}{|c|}{ Velocidade } \\
\cline { 2 - 6 } & DL & DS & Z & US & UL \\
\hline $\mathbf{L}$ & Z & DS & DL & DL & DL \\
\hline $\mathbf{M}$ & US & Z & DS & DL & DL \\
\hline $\mathbf{S}$ & UL & US & Z & DS & DL \\
\hline NZ & UL & UL & Z & DS & DS \\
\hline
\end{tabular}

\subsection{Simulação}

Os modelos matemáticos adotados foram implementados no ambiente MATLAB/Simulink utilizando-se, sempre que possível, os blocos já existentes no Aerospace Blockset (MathWorks, 2010a) e um Personnel Launch System (PLS) também conhecido como HL-20. A missão do HL-20 é transportar pessoas e pequenas quantidades de carga em baixa órbita, utilizando um pequeno sistema de propulsão. Embora o programa HL-20 não esteja ativo atualmente, os projetos da NASA utilizam os dados aerodinâmicos dos testes do HL-20 para novas pesquisas (Stone e Piland, 1991).

O sistema fuzzy também foi implementado no ambiente MATLAB/Simulink. Foi utilizado como base para nossa implementação a Fuzzy Logic Toolbox (MathWorks, 2010b) que permite modelar o comportamento de um sistema complexo utilizando regras lógicas simples e implementar essas regras em um sistema de inferência fuzzy, que para este projeto, foi acoplado aos blocos do Aerospace Blockset. 


\subsection{Modelagem}

Sistemas de orientação de vôo foram considerados como sendo distintos de outros sistemas em uma aeronave. Reconhece-se que em projetos complexos, as funções de orientação de vôo são estreitamente integradas com outras funções do avião, e que a integração física desses sistemas, pode ter uma influência direta na segurança do avião.

No processo de desenvolvimento de um sistema de orientação de aeronaves, uma série de pilotos automáticos são projetados para condições especificas de vôo fornecendo controle parcial ou total da aeronave. Um piloto automático é responsável por uma tarefa e não por toda a missão. O sistema de orientação é responsável por gerenciar a correta ativação e desativação de cada piloto automático em suas fases determinadas. Como mostrado na Figura 6, o subsistema de orientação aceita como entrada o estado atual da aeronave através do Attitude Heading Reference System (AHRS), Air Data System (ADS), Flight Management System (FMS), e Navigation Radios. Usando essa informação, ele calcula os valores que são fornecidos para o piloto automático que consequentemente provê o controle específico da aeronave (Miller et al., 2003).

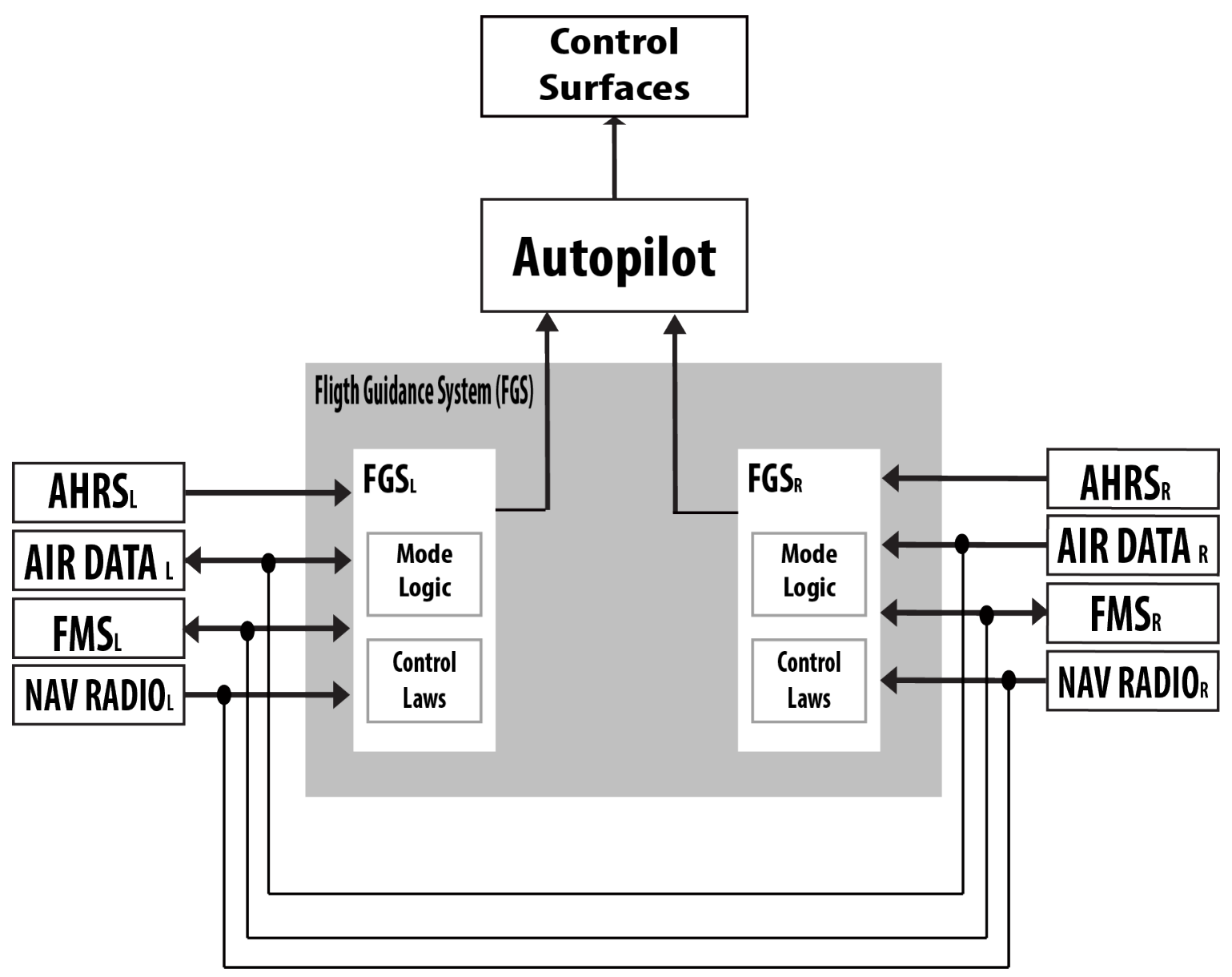

Figura 6. Visão geral do sistema de controle de vôo

Nossa modelagem consiste em substituir a trajetória pré-determinada do glide slope por um modelo fuzzy, como demonstrado na Figura 7, capaz de processar em tempo real os dados de saída necessários para uma correta aterrissagem. 


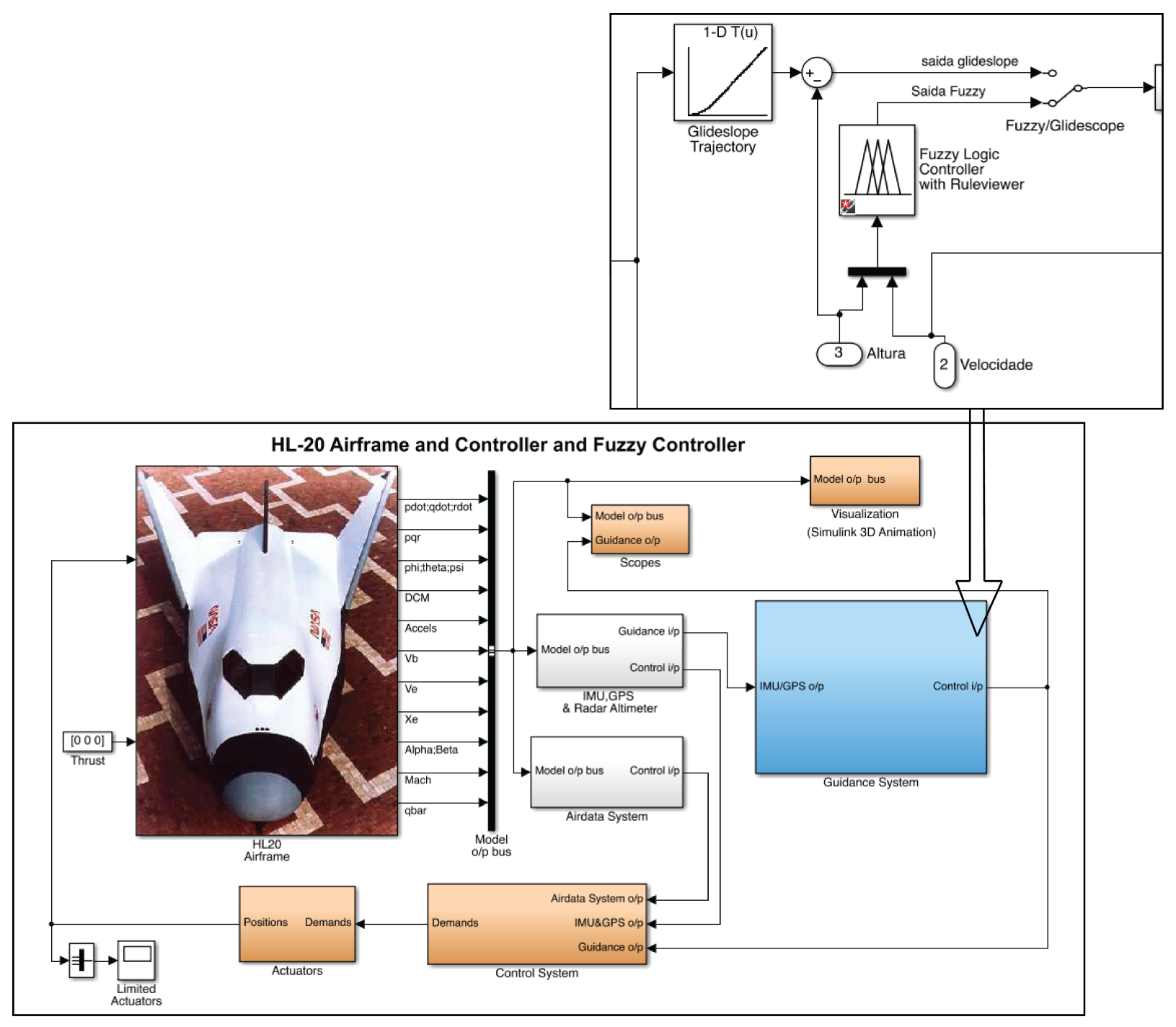

Figura 7. Controlador de aterrissagem com lógica fuzzy

\section{RESULTADOS}

Consideramos um PLS a partir da fase de espera a $1000 \mathrm{~m}$ de altitude em uma velocidade inicial de $62 \mathrm{~m} / \mathrm{s}$. A partir de combinações entre os modelos de agregação na fuzzificação e desfuzzificação do nosso sistema de inferência fuzzy utilizamos um modelo de turbulência, também conhecido como rajadas de Dryden, que é um modelo matemático de rajadas contínuas de vento. O modelo de Dryden, em homenagem a Hugh Dryden, é um dos modelos mais utilizados de rajadas contínuas. Foi publicado pela primeira vez por Liepmann (1952). O modelo Dryden utiliza uma representação espectral para adicionar turbulência para o modelo aeroespacial, fornecendo um ruído branco através de vários filtros. Este modelo é implementado pelo Aerospace Blockset e utiliza a representação matemática da especificação militar americana MILF-8785C e do manual militar MIL-HDBK-1797 (MIL-HDBK-1797, 1997; MIL-F-8785C, 1980). Segundo MathWorks (2010a), turbulência é um processo estocástico definido pelos espectros de velocidade. Para uma aeronave voando a uma velocidade $V$ através de um campo de turbulência com uma frequência espacial de $\Omega$ radianos por metro, a freqüência circular $\omega$ é calculada multiplicando $V$ por $\Omega$. A partir de $2000 \mathrm{~m}$, a intensidade da turbulência é determinada a partir de uma tabela de consulta, que dá a intensidade da turbulência em função da altitude e da probabilidade de excedência na intensidade da turbulência.

Consideramos uma aeronave que consegue concluir a fase de arredondamento como resultado ideal para nossa simulação. Utilizamos três níveis de turbulência, leve, média e alta para cada combinação de agregação do nosso sistema de inferência. Ao todo, foram feitas vinte e sete simulações com $66.6 \%$ de sucesso e $33.4 \%$ de falhas nas quais $11.1 \%$ decorre de um arredondamento mal executado, $14.8 \%$ não pode ser verificado por descontinuidades em variáveis de estado (zero-crossing detection) e $7.4 \%$ foi ocasionado por uma súbita arremetida da aeronave causando a queda da mesma.

De maneira mais especifica, entre as nove combinações feitas a Tabela 2 ilustra os resultados obtidos nas três simulações de turbulência:

Comparamos o nosso modelo com um modelo existente no Simulink, o glideslope trajectory que utiliza valores pré- 
Tabela 2. Aterrissagens bem sucedidas em turbulências leves, média e altas

\begin{tabular}{|c|c|c|c|}
\hline & Max & Probabilistico & Sum \\
\hline Bisector & $66.6 \%$ & $100 \%$ & $66.6 \%$ \\
\hline COA & $66.6 \%$ & $100 \%$ & $66.6 \%$ \\
\hline MOM & $0 \%$ & $66.6 \%$ & $66.6 \%$ \\
\hline
\end{tabular}

determinados em uma matriz n-dimensional para efetuar a aterrissagem. Os resultados obtidos com este modelo nos deram uma base de comparação para o nosso modelo. Efetuando os mesmos testes de turbulência, tivemos resultados positivos para leve e alta turbulência, o que nos deu uma margem de $66.6 \%$ de sucesso. Logo, duas instâncias do nosso sistema se mostraram bastante promissoras: a fuzzificação probabilística e as desfuzzificações bisector e centro de área (COA), que permitiram uma boa aproximação e arredondamento da aeronave em todos os três níveis de turbulência como demonstrado na Figura 8.

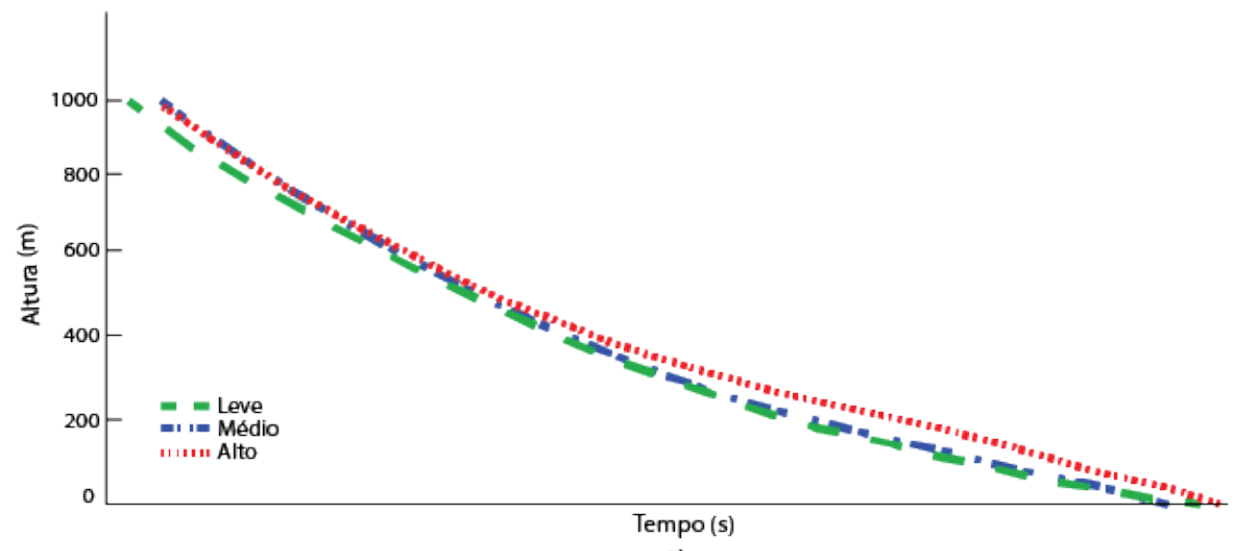

a)

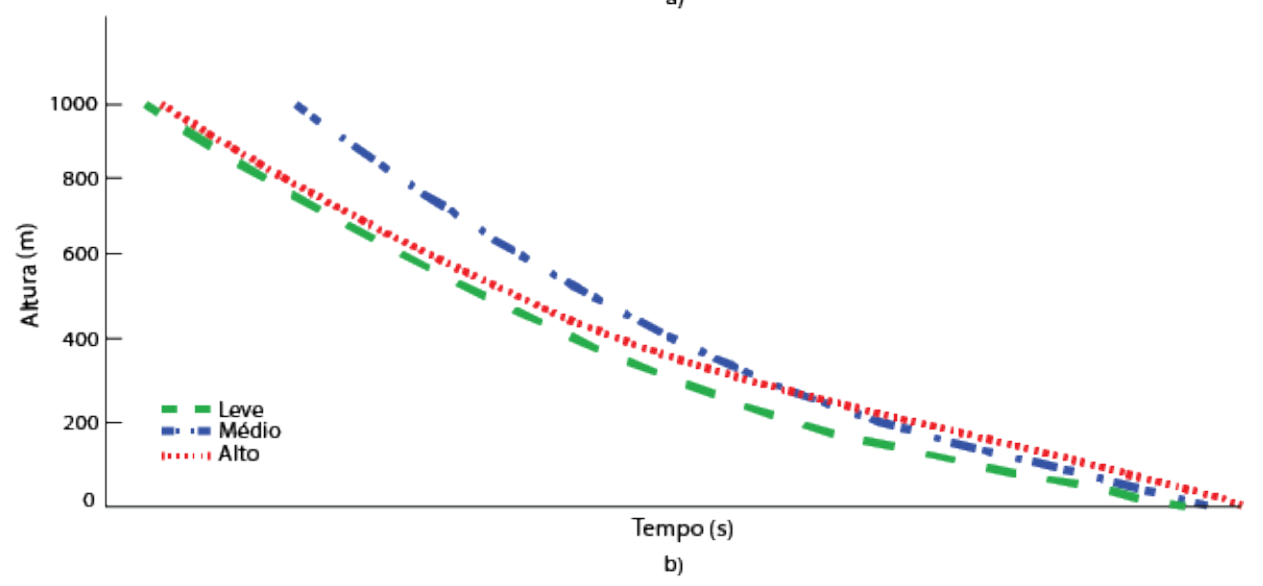

Figura 8. Variação de altura para a) Probabilístico + COA e b) Probabilístico + Bisector

\section{CONCLUSÕES}

Ao ser definido um problema para utilizar um sistema fuzzy, é necessário passar pelas etapas de fuzzificação, inferência e defuzzificação. Conforme aumentamos a quantidade de váriáveis linguísticas, aumentamos então a complexidade do problema em lógica fuzzy, visto que são necessárias regras mais complexas para que o sistema possa gerar uma saída de acordo com o que se deseja. A abrangência dos sistemas fuzzy faz com que ela possa ser empregada em qualquer sistema, alguns até mais recomendados que outros, como por exemplo sistemas de decisão, visto que a capacidade de processamento fuzzy é capaz de fornecer resultados mais próximos ao conhecimento humano.

Os ALS utilizando uma heurística fuzzy adequada, mostraram grande potencial de sucesso em suas aterrissagens, pois inicialmente podemos descartar o fator visão, pois considerando que todo o sistema é baseado nos sensores presentes no ILS, baixa visibilidade não se mostra um problema tangível. Também vimos que o sistema se comportou de maneira adequada em simulações de leve, média e alta turbulência, que inevitavelmente devem ser enfrentadas em um voo real, visto que para a fase de aterrissagem a aeronave necessita obrigatóriamente em algum momento pousar, ao contrário da fase de decolagem o qual em más condições (visibilidade, climática, etc) pode ser cancelada.

De fato um sistema de inferência fuzzy se mostra robusto o suficiente para tratar situações adversas, e como não depende do fator humano, consequentemente não sofre das limitações do mesmo. Logo, a performance ansiada para 
este projeto foi satisfeita, pois o sistema se mostrou completamente funcional. No entanto, não podemos afirmar que nosso sistema não possui margem de erro significativa, considerando que a simulação englobou apenas as funcionalidades básicas de um PLS. As variáveis utilizadas para teste (níveis de turbulência e visibilidade) não são todos os fatores decisivos para uma simulação precisa. Como futura proposta de pesquisa, desejamos contemplar uma quantidade mais relevante de fatores decisivos para simulação, como detecção de falhas, correção em casos de súbitas arremetidas, entre outras.

\title{
REFERÊNCIAS
}

Behera, L., e Kar, I. 2009. Intelligent Systems and Control: Principles and Applications. Oxford University Press.

Boeing, C. A. 2013. Statistical summary of commercial jet statistical summary of commercial jet airplane accidents. Seattle, Washington.

Boskoski, P., Mileva, B., e Deskoski, S. 2005. Auto landing using fuzzy logic. 6th International PhD Workshop on Systems and Control. Izola, Slovenia.

Fuller, J. 2012. SAFETY WAS NO ACCIDENT: History of the UK Civil Aviation Flying Unit CAFU 1944 -1996. Trafford Publishing.

Jang, J., Sun, C., e Mizutani, E. 1997. Neuro-fuzzy and soft computing: a computational approach to learning and machine intelligence. . Prentice Hall.

Lakovic, E., e Lotinac, D. 2010. Aircraft landing control using fuzzy logic and neural networks. IRCSE. Mälardalen University, Sweden.

Liepmann, H. W. 1952. On the application of statistical concepts to the buffeting problem. Journal of the Aeronautical Sciences, 19(12), 793-800.

MathWorks, I. 2010a. Aerospace Blockset, User's Guide.

MathWorks, I. 2010b. Fuzzy Logic Toolbox, User's Guide.

McLauchlan, L. L. L. 2009. Fuzzy logic controlled landing of a boeing 747. IEEE/RSJ International Conference on Intelligent Robots and Systems. St. Louis, USA.

MIL-F-8785C 1980. Flying Qualities of Piloted Airplanes. USA: Department of Defense.

MIL-HDBK-1797 1997. Flying Qualities of Piloted Aircraft. USA: Department of Defense.

Miller, S. P., Tribble, A. C., Carlson, T. M., e Danielson, E. J. 2003. Flight guidance system requirements specification. National Aeronautics and Space Administration.

Paglione, P. 1985. Desempenho de aeronaves: Fundamentos, condições de voo e segmentos de trajetória. São José dos Campos: ITA.

Raj, K. D. S., e Tattikota, G. 2013. Design of fuzzy logic controller for auto landing applications. International Journal of Scientific and Research Publications, 3, 536.

Stone, H., e Piland, W. 1991. The hl-20 lifting-body personnel launch system. SAE Technical Paper, (911970).

Zadeh, L. A. 1965. Fuzzy sets. Information and Control, 8(3), 338-353.

\section{RESPONSABILIDADE AUTORAL}

As opiniões, hipóteses e conclusões ou recomendações expressas neste material são de responsabilidade exclusiva dos autores.

\section{Study and development of simulators for landing aircraft using fuzzy logic.}

\author{
Rafael de Oliveira Taveira, rottaveira@gmail.com ${ }^{1}$ \\ Sergio Francisco da Silva, sergio.f. silva@gmail. com $^{1}$
}

${ }^{1}$ Universidade Federal de Goiás, Av. Dr. Lamartine Pinto de Avelar, 1120, St.Universitário, Catalão - GO

\begin{abstract}
The notion of fuzzy sets it was conceived in 1965, the focus was to establish a way of treating problems of subjective nature with vague and imprecise information in a formal way and also deal with problems where there are numerical data and knowledge in linguistic form. Based on the theory of fuzzy logic, this paper aims to present an automatic landing system for aircraft, which can be termed as a way to guide and control an aircraft automatically from an initial estimation of altitude for a point where a secure contact is made with the landing surface. These systems provide not only guidance but also provide control of the aircraft, including information about the speed and position of the aircraft relative to the ground below it.
\end{abstract}

Keywords: fuzzy, airplane, landing 